\title{
MODERNIZACIÓN DEL SISTEMA EDUCATIVO: \\ LA TENSIÓN ENTRE TRANSFORMAR Y CONSERVAR
}

MARGARITA MARÍA ZORRILLA FIERRO

Departamento de Educación/UAA

Salvador Camacho Sandoval,

Modernización Educativa en México, 1992-1998. El Caso de Aguascalientes, Aguascalientes, UAA-IEA, 2002.

Suele decirse que los libros se leen "acabados", ya que difícilmente los lectores tienen acceso al proceso de construcción y producción de los autores, pero en esta ocasión puedo decir que no es mi caso, pues conozco el origen de lo que Salvador Camacho expone en su libro. Además, tuve la oportunidad de estar en el ojo del huracán de la reforma educativa en Aguascalientes. Ambas cuestio nes, conocer y haber tenido la experiencia, me colocan en una tesitura especial frente a este libro. A continuación se exponen algunos puntos que me resultaron de importancia.

\section{EL CONTENIDO DEL LIBRO}

En la parte introductoria, Salvador Camacho expone aquellos elementos que consideró pertinentes para ubicar la naturaleza del libro en cuanto a su contenido y metodología. Asimismo, explicita los propósitos del trabajo que enunciados de manera breve son:

- Analizar históricamente la reforma educativa iniciada en el gobierno de Miguel de la Madrid en el marco del proyec- 
to socioeconómico instrumentado y a la luz de los cambios en los sistemas educativos.

- Analizar la aplicación de la reforma educativa modernizadora en Aguascalientes para identificar la manera cómo la política educativa respondió a las demandas del desarrollo socioeconómico.

- Comparar la relación entre la reforma educativa mexicana y la ideología modernizadora (neoliberal).

Como podemos advertir, los propósitos son ambiciosos por su complejidad y amplitud. La organización del libro permite incursionar sobre el devenir histórico de la educación en México y en Aguascalientes; enseguida plantea experiencias de reforma educativa en otros países y en particular cómo es que México se inserta en los procesos de transformación de los sistemas educativos. En tercer lugar se describe a Aguascalientes tanto por lo que se refiere a las características de su desarrollo socioeconómico como de las medidas educativas instrumentadas en el estado hasta antes de 1992.

En el cuarto capítulo narra el autor las resistencias presentadas a las propuestas de reforma a partir de 1992 y en un quinto apartado busca describir la instrumentación de la política educativa modernizadora en Aguascalientes en el periodo 1992-1998, para concluir con un análisis que tiene como propósito integrar los elementos antes expuestos.

\section{LA TESIS DEL AUTOR}

Neoliberalismo, globalización y modernización son tres términos que de unos años a la fecha suelen estar presentes en múltiples conversaciones entre académicos de distinta denominación, como son los economistas, politólogos, sociólogos, educadores o de los mismos políticos. Cada quien los lee desde sus peculiares modos de ver el mundo y el devenir de las sociedades. Se definen, se problematizan, se critican los procesos sociales que generan y, sin embargo, en educación creo que aún no alcanzamos a ver con claridad sus implicaciones.

Salvador Camacho nos ofrece en el primer capítulo de su libro una serie de consideraciones teóricas acerca de los términos antes aludidos. Su capacidad para explicar lo que acontece en 
la terca realidad, es variable e incluso limitada. Por el tema general del libro, que remite fundamentalmente a la definición e implementación de políticas públicas en educación, quiero recuperar una cita de Olac Fuentes Molinar.

La política gubernamental no se concibe como el desenvolvimiento lineal de un plan maestro, sino como búsqueda de estrategias y formas de intervención política, que parten ciertamente de juicios e intenciones de la cúpula de la burocracia gubernamental, pero no de formulaciones rígidas y prediseñadas. Así, la concreción de la política va respondiendo, en sus formas y sus tiempos, a las respuestas de los actores involucrados y a los efectos no previstos que la propia política va generando. Desde esta perspectiva, es natural la provisionalidad de muchas de las formulaciones y medidas políticas, las rectificaciones, la interrupción de iniciativas y la incorporación no previsible de nuevas propuestas. Esta cita expresa la tesis central del libro:

La instrumentación de la política educativa modernizadora en México se ha desarrollado de una manera zigzagueante y no exenta de contradicciones, lo cual permite suponer que sus vínculos con el conjunto de las políticas 'neoliberales' no son tan claros y determinantes como suele suponerse $[\ldots]$.

Me pregunto, siguiendo a Olac Fuentes: ¿Acaso puede ser de otra manera? Pienso que no. Quizá el tema sería preguntarnos acerca de cómo los sistemas educativos y quienes los conducen enfrentan y van solucionando las contradicciones.

En este sentido yo haría una lectura distinta de los acontecimientos teniendo como base una perspectiva histórica que propiciara la comprensión de cómo es que se fueron resolviendo o no, las resistencias en cuya base están instalados los intereses de poder de distintos actores.

El otro tema, que de alguna manera Salvador Camacho expone en su libro, es el que se refiere al concierto internacional caracterizado por la llamada globalización. Aquí la pregunta sería: ¿Quién o quiénes definen las políticas educativas? ¿Podemos hablar de originalidad en el caso mexicano? ¿En qué sentido la política educativa mexicana no es más que una expresión de políticas definidas en el concierto internacional en el cual 
intervienen distinto tipo de organismos económicos y financieros? ¿Cómo mostrar empíricamente estas relaciones? Sin duda, la historia es un acercamiento posible.

TRANSFORMAR EL SISTEMA EDUCATIVO MEXICANO

Del complejo y dinámico mundo de la educación nacional, se presentan cuatro comentarios.

1. La tensión entre transformar y conservar es una peculiaridad de los sistemas educativos, pues la educación por un lado tiene la misión de conservar la cultura y transmitirla a las nuevas generaciones y, al mismo tiempo, acrecentarla y transformarla. Esta situación de facto, dicha así, parece sencilla, pero no lo es. Esta misión se complica por el funcionamiento del sistema educativo desde al nivel jerárquico más alto que es la Secretaría de Educación Pública, hasta el salón de clases de cualquier tipo y nivel educativo. ¿Qué permanece, qué cambia y por qué? es una pregunta básica que les haría a los historiadores?

2. La vinculación entre educación y desarrollo, al menos en el discurso, siempre ha estado presente. Salvador Camacho da cuenta de ello de distinta manera.

Sin embargo, no disponemos de elementos suficientes que nos permitan afirmar en qué grado en México la educación ha contribuido al desarrollo. Lo que si me parece claro es que las decisiones en educación van después de las definiciones de política económica. Me pregunto si podría ser de otra manera.

3. El proyecto modernizador y la educación. Continuando con el argumento anterior, lo que ahora les está sucediendo a muchos sistemas educativos en el mundo, pero de manera especial a México y a los países de América Latina y el Caribe, es el de recibir las exigencias de un Estado que dejó de ser de Bienestar para ser un Estado-Gobierno que admi-nistra y regula. Claro que este proceso de transformación del Estado no está concluido, digamos que se presenta en fase de transición.

Esta realidad tiene implicaciones en la reforma del sistema educativo mexicano expresada en las políticas y programas que de ella se deriven. La expresión más clara del proyecto modernizador en el caso del sistema educativo es la descentralización o federalización descentralizadora como diría Alberto Arnaut.

4. En su análisis del sistema 
educativo mexicano, el autor enfatiza las dificultades para mejorar la educación y señala que éstas están "relacionadas con factores de carácter político y sindical". Tres comentarios. Uno tiene que ver con los factores político y sindical, sobre los cuáles habría que profundizar más para identificar su naturaleza y mecanismos de funcionamiento. Dos, ya se sabe por otras investigaciones que además de estos factores están los que hacen referencia a la dimensión técnico profesional, cultural y pedagógica que interactúan entre sí de distinta manera. Y tres, el enfoque de obstáculos y dificultades es sólo una vía para el análisis, a la cual yo añadiría la que se refiere a las fuerzas o factores que posibilitan ciertos cambios.

\section{Y Aguascalientes, ¿̇ouÉ?}

Empezaría diciendo que disiento de la manera en que el autor inicia el apartado. "Aguascalientes: laboratorio de reformas". Creo que en sentido estricto no corresponde este sustantivo. Por razones que desconozco y que habría que averiguar en la historia, esta denominación de Aguascalientes como un laboratorio de reformas, ha estado presente en las conversaciones o en declaraciones de los políticos. Pero, ¿qué significa esto? Se supone que por el tamaño del estado sería factible introducir "innovaciones" para observar qué sucede, aprender del experimento y tomar medidas para escalar la experiencia a otras dimensiones. En la práctica, en el sistema educativo real, no suelen suceder las cosas así. Al menos en los últimos quince años se define una acción de política y se implanta sin mayores pruebas previas.

En este sentido, cuando se decide federalizar el sistema educativo, en su significado descentralizador, la medida se aplica en todos los estados, sin que el caso Aguascalientes haya sido tratado en forma especial. Si nuestro estado fue una excepción, ello se debe a que el gobierno de Otto Granados fue bastante agresivo en materia educativa, lo que propició que fueran creándose condiciones políticas y sindicales, así como técnico-profesionales, para definir distintos componentes de lo que en el estado podría ser la reforma educativa. Al igual que en Aguascalientes, en otros nueve estados coincide la firma del Acuerdo Nacional para la Moder-

D


nización de la Educación Básica con nuevos gobiernos estatales; sin embargo, el impulso modernizador no se dio de la misma manera, pues las peculiaridades de cada estado fueron definiendo por acción u omisión la nueva conducción de sus sistemas educativos.

Salvador Camacho narra algunas cuestiones que estuvieron en el ambiente en los años previos a la descentralización, que pueden ser vistas como caldo de cultivo para los cambios. En particular, refiere acontecimientos del primer año de la reforma educativa que no agotan los procesos que ocurrieron. Por ejemplo, poco se conoce acerca de cómo se elaboró el Plan de Desarrollo 1992 1998. Dos equipos distintos, la Comisión de Educación y otro que después se convertiría en la Oficina de Asesores del Gobernador tuvieron la encomienda de elaborar el capítulo referente a la educación. Aquí sería importante destacar la disputa silenciosa que tuvo lugar entre agosto y noviembre de 1992. Finalmente se impuso el documento elaborado en la Comisión de Educación como texto del Programa Estatal de Educación, del cual se conocie- ron después diferentes versiones. Disiento de la afirmación del autor acerca de que dicho Programa carecía de una estructura programática coherente en sus objetivos, metas y acciones y que era más bien una expresión de anhelos y aspiraciones. Por lo que conozco y por la intervención que tuve en estos procesos, se lograron definir ocho objetivos estratégicos que mostraban con bastante claridad las cosas que habría que atender en el periodo gubernamental. La falta de una especificación de metas en términos de "compromisos gubernamentales" es real, pero lo mismo pasó con los programas de los otros sectores. ¿A qué se debió esta omisión? Sería bueno indagarlo. No obstante las deficiencias técnicas en el nivel programático de las primeras versiones de la política educativa, los objetivos permitieron darle una coherencia a los proyectos y acciones que de ellos se fueron derivando.

El libro enfatiza, para el caso de Aguascalientes, los obstáculos y dificultades que enfrentó el nuevo gobierno. Habría que señalar que la acción gubernamental en materia educativa fue logrando una creciente legitimidad y credibilidad entre la población y 
entre los maestros. Mi hipótesis es que el nivel de las negociaciones políticas entre grupos de interés tuvieron éxito y se facilitó la introducción de nuevas formas de organización y acción en el sistema educativo local.

En particular, como lo señala el autor, el tema de la reforma organizativa de la supervisión escolar, tocó fibras sensibles del sistema y de alguna manera inició un proceso que trastocó las viejas prácticas y tal vez esto fue posible por una condición política: tanto los supervisores como el nuevo gobierno eran priístas. Se tienen datos de que en estados donde el gobierno es de un signo partidista distinto, la confrontación con los supervisores fue la característica dominante. De hecho, aún en medio de las manifestaciones que tuvieron lugar en mayo de 1993, no se detuvieron los trabajos que se estaban realizando al interior del Instituto de Educación de Aguascalientes.

¿Hasta dónde las resistencias que se expresaron en Aguascalientes fueron expresión de condiciones locales o más bien se alimentaron como una manifestación de los disensos que tenía Elba Esther Gordillo, entonces líder del SNTE, respecto a la federalización? Hasta donde sabemos, y así lo señala el autor, el presidente Carlos Salinas fue quien directamente paró el movimiento.

Salvador Camacho menciona otros elementos de la política educativa, como la Ley de Educación del Estado, la cual merece atención especial. También menciona otros puntos importantes como los referidos a la educación media, la educación superior y la ciencia y la tecnología. Sobre todo esto, el autor contribuye a responder preguntas como las siguientes: ¿Cuáles fueron los alcances de cada política? ¿Cuál fue el saldo de la gestión gubernamental 1992-1998?

\section{PARA TERMINAR}

Documentar y explicar el acontecer, en este caso del sistema educativo, en una perspectiva nacional (o central) y una local es importante, porque contribuye a construir una perspectiva dinámica de una entidad, que como el sistema educativo es un organismo vivo.

Las perspectivas teóricas y conceptuales elegidas para mirar dicho acontecer sin duda nos permiten ver unas cosas y no otras y de esto tenemos que 
hacernos concientes. Es decir, tendremos información parcial de un objeto complejo y dinámico.

¿Cómo se relacionan globalización, modernización y neoliberalismo con las políticas y programas educativos? ¿En qué niveles de la estructura del sistema educativo se manifiestan en caso de estar relacionados? ¿Les sucede algo a la escuela y a los procesos de enseñanza y aprendizaje que en ella acon- tecen? Son preguntas para las que todavía no tenemos respuestas completamente satisfactorias, por lo que libros como el de Salvador Camacho son bienvenidos, pues alientan la búsqueda de una mejor comprensión del sistema educativo.

Al mismo tiempo, estas respuestas parciales pueden ayudar a actuar de mejor manera y tener como resultado una mejor educación. 